\title{
Finite-Difference Frequency-Domain Analysis of 2-D Photonic Crystals With Curved Dielectric Interfaces
}

\author{
Yen-Chung Chiang, Member, IEEE, Yih-Peng Chiou, Member, IEEE, and Hung-Chun Chang, Senior Member, IEEE
}

\begin{abstract}
An improved finite-difference frequency-domain method based on Taylor series expansion, local coordinate transformation, and boundary condition matching is developed for analyzing the band diagrams of 2-D photonic crystals. This newly proposed scheme can deal with piecewise homogeneous structures with curved dielectric interfaces. It also shows much better performance in modeling structures of highly index contrast ratio. We have verified this improved scheme with frequently-used plane-wave expansion method through the calculation of the band diagrams of a 2-D photonic crystal with square lattice. We also compare the scheme with the staircase approximation. We further improve the convergence behavior by adopting high-order terms. The result of this adopting is also demonstrated.
\end{abstract}

Index Terms-Electromagnetic propagation, finite-difference methods, frequency-domain analysis, photonic crystals, step-index waveguides.

\section{INTRODUCTION}

I $\mathrm{N}$ THE LAST two decades a new frontier in materials suggests that we might be able to tailor the properties of light propagation just as we do in semiconductor devices. The key of achieving this goal lies in the use of a new class of material called photonic crystals. These new categories of materials are characterized by their photonic band gaps in which waves are forbidden to propagate for some frequencies due to the periodicity in the structures [1]-[7]. We may design various devices based on this characteristic by properly introducing defects between the periodic structures. Thus, the calculation of the band diagram is fundamental for analyzing the photonic crystals and the design of devices.

Many numerical methods have been proposed for the calculation of the band structures of photonic crystals. Among these the most commonly used methods are the plane-wave expansion method [3]-[5] and the finite-difference time-domain (FDTD)

Manuscript received August 25, 2007; revised December 10, 2007. This work was supported in part by the National Science Council of the Republic of China under Grant NSC 94-2218-E-005-016 and Grant NSC 95-2221-E-005-127 and by the Ministry of Education, Taiwan, under the ATU plan.

Y.-C. Chiang is with the Department of Electrical Engineering, National Chung-Hsing University, Taichung 402-27, Taiwan, R.O.C. (e-mail: ycchiang1970@nchu.edu.tw).

Y.-P. Chiou is with the Graduate Institute of Photonics and Optoelectronics and Department of Electrical Engineering, National Taiwan University, Taipei 106-17, Taiwan, R.O.C. (e-mail: ypchiou@ntu.edu.tw).

H.-C. Chang is with the Department of Electrical Engineering, the Graduate Institute of Photonics and Optoelectronics, and the Graduate Institute of Communication Engineering, National Taiwan University, Taipei 106-17, Taiwan, R.O.C. (e-mail: hcchang@cc.ee.ntu.edu.tw).

Digital Object Identifier 10.1109/JLT.2007.916499 method [6], [7]. In the plane-wave expansion method, the frequencies (eigenvalues) and its corresponding fields (eigenvectors) can be obtained via an iterative process, but the resultant matrix is a large and dense one. Furthermore, it shows slow convergence due to the abrupt discontinuities of fields or field derivatives. In the FDTD method, the resonant modes in the bands are obtained from the frequency analysis by imposing periodic boundary condition in a unit cell. However, the resonant frequencies may be missed if the excitation or monitor is not properly placed. In addition, it has limitation in resolving degenerate modes. Aside from the plane-wave expansion method and FDTD method, finite-difference frequency-domain approaches for analyzing the band structures of 2-D photonic crystals have also been proposed due to its sparsity and efficiency. Yang [8] proposed a finite-difference frequency-domain scheme based on directly discretizing the Helmholtz's equation in the homogeneous mesh region and matching the field at the central grid point. Shen et al. [9] derived another finite-difference scheme with the effective medium technique. Both methods are suitable only for irregular structures with interfaces parallel to the axes but not for those with curved shapes.

As for the curved dielectric interface in the structures, conventional finite-difference methods generally adopt either the staircase index [10], [11], the graded-index [12], or the average index [13] approximation. These approximations may be good enough for the weakly guiding structures, but not for the strongly guiding ones which are common in photonic crystal structures. Some improved finite-difference schemes [14]-[16] have been proposed to deal with the step-index interface in one-dimensional problems, but these methods fail to deal with 2-D curved dielectric interface such as linear slanted or curved interfaces commonly found in photonic crystals. Some finite-difference schemes have been proposed for the slanted step-index interfaces [17], but they still require index-average assumption or interpolation in geometry in those schemes. In [18], we have proposed a new finite-difference scheme that can successfully deal with 2-D curved step-index interfaces for optical waveguides. However, the proposed method is based on the transverse fields and is only suitable for out-of-plane propagation. To make the formulas complete, those for in-plane propagation are needed as well. In this paper, an improved finite-difference scheme for in-plane propagation is derived to analyze the band structures of 2-D photonic crystals with curved step-index interfaces. Since we adopted a rigorous field expansion technique in our derivation, the only error of our formulas comes from the truncation of the higher-order terms. 


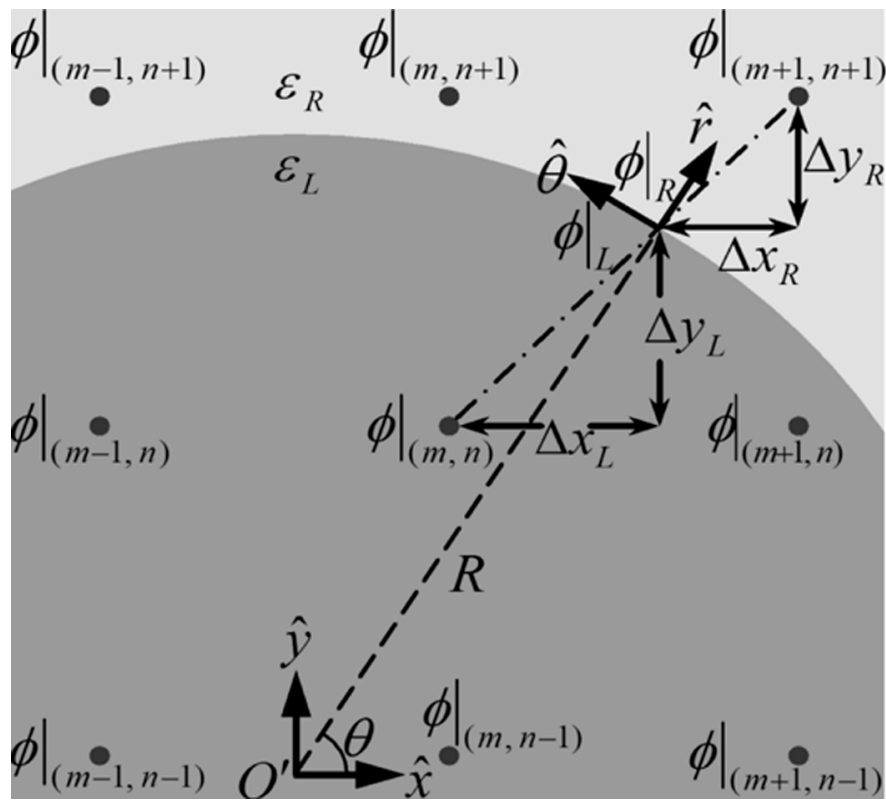

Fig. 1. Cross section of general finite-difference mesh with a curved interface.

Thus, it is easy to derive the high-order formulas following the same processes.

The detailed derivation of our formulas is given in Section II. Some numerical results are presented in Section III including the comparison of our method with others. A simple conclusion is drawn in Section IV.

\section{FORMULATION}

The cross section under consideration is shown in Fig. 1. We will derive a general relation between the field $\left.\phi\right|_{(m, n)}$ at the sampled point $(m, n)$ and the fields at the nearby points with a curved interface in-between. The quantity $\phi$ denotes $E_{z}$ for TM modes or $H_{z}$ for TE modes at the sampled point. We define a local cylindrical coordinate system originating at $O^{\prime}$ by the normal vector $\hat{r}$ and the tangential vectors $\hat{\theta}$. The effective radius is $R$. The procedures to derive our formulas are similar to that which we demonstrated in [18]. We first express the fields at surrounding points as a linear combination of the field at the center node and its derivatives. Take the relation between $\left.\phi\right|_{(m+1, n+1)}$ and $\left.\phi\right|_{(m, n)}$ as an example, the procedures can be summarized as follows:

1) express the field $\left.\phi\right|_{(m+1, n+1)}$ as the a 2-D Taylor series expansion of the field just right to the interface, $\left.\phi\right|_{R}$;

2) transform $\left.\phi\right|_{R}$ and its derivatives in the globe $x-y$ coordinates system into the local $r-\theta$ coordinates system;

3) express $\left.\phi\right|_{R}$ and its derivatives as a linear combination of the field just left to the interface, $\left.\phi\right|_{L}$, and its derivatives by matching the boundary conditions;

4) transform $\left.\phi\right|_{L}$ and its derivatives in the $r-\theta$ coordinates system back to the $x-y$ coordinates system;

5) express $\left.\phi\right|_{L}$ and its derivatives as a 2-D Taylor series expansion of $\left.\phi\right|_{(m, n)}$ and its derivatives.

Following the previous five steps, we can express $\left.\phi\right|_{(m+1, n+1)}$ as the linear combination of $\left.\phi\right|_{(m, n)}$ and its derivatives. We will explain each step in detail in the following subsections.

\section{A. Taylor Series Expansion}

The problem under consideration is piecewise homogeneous, and we can employ the 2-D Taylor series expansion within the homogeneous region. For example, assuming that the $x$ - and $y$-displacements from the point $(m+1, n+1)$ to the interface are $\Delta x_{R}$ and $\Delta y_{R}$ as shown in Fig. 1, we can express the field quantity $\left.\phi\right|_{(m+1, n+1)}$ as the Taylor series expansion of the field just right to the interface, $\left.\phi\right|_{R}$, and its derivatives

$$
\begin{array}{r}
\left.\phi\right|_{(m+1, n+1)}=\left.\phi\right|_{R}+\left.\Delta x_{R} \frac{\partial \phi}{\partial x}\right|_{R}+\left.\Delta y_{R} \frac{\partial \phi}{\partial y}\right|_{R}+\left.\frac{\Delta x_{R}^{2}}{2 !} \frac{\partial^{2} \phi}{\partial x^{2}}\right|_{R} \\
+\left.\frac{2 \Delta x_{R} \Delta y_{R}}{2 !} \frac{\partial^{2} \phi}{\partial x \partial y}\right|_{R}+\left.\frac{\Delta y_{R}^{2}}{2 !} \frac{\partial^{2} \phi}{\partial y^{2}}\right|_{R}+\cdots \quad \text { (1) }
\end{array}
$$

Similarly, we can express the field quantity at just left to the interface, $\left.\phi\right|_{L}$, as the 2-D Taylor series expansion of the field, $\left.\phi\right|_{(m, n)}$, and its derivatives

$$
\begin{aligned}
\left.\phi\right|_{L}= & \left.\phi\right|_{(m, n)}+\left.\Delta x_{L} \frac{\partial \phi}{\partial x}\right|_{(m, n)}+\left.\Delta y_{L} \frac{\partial \phi}{\partial y}\right|_{(m, n)}+\left.\frac{\Delta x_{L}^{2}}{2 !} \frac{\partial^{2} \phi}{\partial x^{2}}\right|_{(m, n)} \\
& +\left.\frac{2 \Delta x_{L} \Delta y_{L}}{2 !} \frac{\partial^{2} \phi}{\partial x \partial y}\right|_{(m, n)}+\left.\frac{\Delta y_{L}^{2}}{2 !} \frac{\partial^{2} \phi}{\partial y^{2}}\right|_{(m, n)}+\cdots \quad(2)
\end{aligned}
$$

where $\Delta x_{L}$ and $\Delta y_{L}$ are the $x$ - and $y$-displacements from the interface to the point $(m, n)$ as shown in Fig. 1. By differentiating (2) with respect to $x$ and $y$, we have

$$
\begin{gathered}
\left.\frac{\partial \phi}{\partial x}\right|_{L}=\left.\frac{\partial \phi}{\partial x}\right|_{(m, n)}+\left.\Delta x_{L} \frac{\partial^{2} \phi}{\partial x^{2}}\right|_{(m, n)}+\left.\Delta y_{L} \frac{\partial^{2} \phi}{\partial x \partial y}\right|_{(m, n)} \\
+\left.\frac{\Delta x_{L}^{2}}{2 !} \frac{\partial^{3} \phi}{\partial x^{3}}\right|_{(m, n)}+\left.\frac{2 \Delta x_{L} \Delta y_{L}}{2 !} \frac{\partial^{3} \phi}{\partial x^{2} \partial y}\right|_{(m, n)}+\cdots
\end{gathered}
$$

and

$$
\begin{aligned}
& \left.\frac{\partial \phi}{\partial y}\right|_{L}=\left.\frac{\partial \phi}{\partial y}\right|_{(m, n)}+\left.\Delta x_{L} \frac{\partial^{2} \phi}{\partial x \partial y}\right|_{(m, n)}+\left.\Delta y_{L} \frac{\partial^{2} \phi}{\partial y^{2}}\right|_{(m, n)} \\
& +\left.\frac{\Delta x_{L}^{2}}{2 !} \frac{\partial^{3} \phi}{\partial x^{2} \partial y}\right|_{(m, n)}+\left.\frac{2 \Delta x_{L} \Delta y_{L}}{2 !} \frac{\partial^{3} \phi}{\partial x \partial y^{2}}\right|_{(m, n)}+\cdots
\end{aligned}
$$

Similarly, the higher-order derivatives of $\left.\phi\right|_{L}$ can thus be obtained in terms of the derivatives of $\left.\phi\right|_{(m, n)}$ by successively differentiating (3) and (4) with respect to $x$ or $y$.

\section{B. Local Coordinate Transformation}

The field quantities and their derivatives with respect to $x$ or $y$ then need be transformed into the corresponding quantities and derivatives with respect to $r$ or $\theta$ in the local cylindrical coordinate system defined above. The relations to transform from the global $x-y$ coordinate system into the local $r-\theta$ coordinate system are given in the following:

$$
\begin{aligned}
\left.\phi\right|_{x y \text {-coordinate }} & =\left.\phi\right|_{r \theta \text {-coordinate }} \\
\frac{\partial \phi}{\partial x} & =\cos \theta \frac{\partial \phi}{\partial r}-\frac{\sin \theta}{r} \frac{\partial \phi}{\partial \theta} \\
\frac{\partial \phi}{\partial y} & =\sin \theta \frac{\partial \phi}{\partial r}+\frac{\cos \theta}{r} \frac{\partial \phi}{\partial \theta}
\end{aligned}
$$




$$
\begin{aligned}
\frac{\partial^{2} \phi}{\partial x^{2}}= & \frac{\sin ^{2} \theta}{r} \frac{\partial \phi}{\partial r}+\frac{2 \cos \theta \sin \theta}{r^{2}} \frac{\partial \phi}{\partial \theta}+\cos ^{2} \theta \frac{\partial^{2} \phi}{\partial r^{2}} \\
& -\frac{2 \cos \theta \sin \theta}{r} \frac{\partial^{2} \phi}{\partial r \partial \theta}+\frac{\sin ^{2} \theta}{r^{2}} \frac{\partial^{2} \phi}{\partial \theta^{2}} \\
\frac{\partial^{2} \phi}{\partial x \partial y}= & -\frac{\cos \theta \sin \theta}{r} \frac{\partial \phi}{\partial r}+\frac{\cos ^{2} \theta-\sin ^{2} \theta}{r^{2}} \frac{\partial \phi}{\partial \theta} \\
& +\cos \theta \sin \theta \frac{\partial^{2} \phi}{\partial r^{2}}+\frac{\cos ^{2} \theta-\sin ^{2} \theta}{r} \frac{\partial^{2} \phi}{\partial r \partial \theta} \\
& -\frac{\cos \theta \sin \theta}{r^{2}} \frac{\partial^{2} \phi}{\partial \theta^{2}} \\
\frac{\partial^{2} \phi}{\partial y^{2}}= & \frac{\cos ^{2} \theta}{r} \frac{\partial \phi}{\partial r}-\frac{2 \cos \theta \sin \theta}{r^{2}} \frac{\partial \phi}{\partial \theta}+\sin ^{2} \theta \frac{\partial^{2} \phi}{\partial r^{2}} \\
& +\frac{2 \cos \theta \sin \theta}{r} \frac{\partial^{2} \phi}{\partial r \partial \theta}+\frac{\cos ^{2} \theta}{r^{2}} \frac{\partial^{2} \phi}{\partial \theta^{2}} \\
& \vdots
\end{aligned}
$$

We can also transform the quantities from the local $r-\theta$ coordinates system back to the global $x-y$ coordinate system in a similar manner. The relations are given by the following equations:

$$
\begin{aligned}
\frac{\partial \phi}{\partial r}= & \cos \theta \frac{\partial \phi}{\partial x}+\sin \theta \frac{\partial \phi}{\partial y} \\
\frac{\partial \phi}{\partial \theta}= & -r \sin \theta \frac{\partial \phi}{\partial x}+r \cos \theta \frac{\partial \phi}{\partial y} \\
\frac{\partial^{2} \phi}{\partial r^{2}}= & \cos ^{2} \theta \frac{\partial^{2} \phi}{\partial x^{2}}+2 \cos \theta \sin \theta \frac{\partial^{2} \phi}{\partial x \partial y}+\sin ^{2} \theta \frac{\partial^{2} \phi}{\partial y^{2}} \\
\frac{\partial^{2} \phi}{\partial r \partial \theta}= & -\sin \theta \frac{\partial \phi}{\partial x}+\cos \theta \frac{\partial \phi}{\partial y}-r \cos \theta \sin \theta \frac{\partial^{2} \phi}{\partial x^{2}} \\
& +r\left(\cos ^{2} \theta-\sin ^{2} \theta\right) \frac{\partial^{2} \phi}{\partial x \partial y}+r \cos \theta \sin \theta \frac{\partial^{2} \phi}{\partial y^{2}} \\
\frac{\partial^{2} \phi}{\partial \theta^{2}}= & -r \cos \theta \frac{\partial \phi}{\partial x}-r \sin \theta \frac{\partial \phi}{\partial y}+r^{2} \sin { }^{2} \theta \frac{\partial^{2} \phi}{\partial x^{2}} \\
& -2 r^{2} \cos \theta \sin \theta \frac{\partial^{2} \phi}{\partial x \partial y}-r^{2} \cos ^{2} \theta \frac{\partial^{2} \phi}{\partial y^{2}} \\
& \vdots
\end{aligned}
$$

\section{Matching Boundary Conditions}

We then need to link the quantity $\left.\phi\right|_{R}$ and its derivatives at just right to the interface with those at the other side of the interface. We have derived the relations by utilizing proper interface condition matching technique. The boundary conditions that need to be matched are given as follows for the TM modes $\left(\phi=E_{z}\right)$ :

$$
\begin{aligned}
\left.E_{z}\right|_{R} & =\left.E_{z}\right|_{L} \\
\left.\frac{\partial E_{z}}{\partial r}\right|_{R} & =\left.\frac{\partial E_{z}}{\partial r}\right|_{L} \\
\left.\frac{\partial E_{z}}{\partial \theta}\right|_{R} & =\left.\frac{\partial E_{z}}{\partial \theta}\right|_{L} \\
\left.\frac{\partial^{2} E_{z}}{\partial r^{2}}\right|_{R} & =\left.\frac{\partial^{2} E_{z}}{\partial r^{2}}\right|_{L}+\left.k_{0}^{2}\left(\varepsilon_{L}-\varepsilon_{R}\right) E_{z}\right|_{L}
\end{aligned}
$$

$$
\begin{aligned}
& \left.\frac{\partial^{2} E_{z}}{\partial r \partial \theta}\right|_{R}=\left.\frac{\partial^{2} E_{z}}{\partial r \partial \theta}\right|_{L} \\
& \left.\frac{\partial^{2} E_{z}}{\partial \theta^{2}}\right|_{R}=\left.\frac{\partial^{2} E_{z}}{\partial \theta^{2}}\right|_{L}
\end{aligned}
$$

where $\varepsilon_{L}$ and $\varepsilon_{R}$ are the permittivities on the left-hand and righthand sides of the interface, respectively, as shown in Fig. 1, and $k_{0}$ is the free-space wavenumber. Similar equations can also be found for the TE modes $\left(\phi=H_{z}\right)$ as follows:

$$
\begin{aligned}
\left.H_{z}\right|_{R}= & \left.H_{z}\right|_{L} \\
\left.\frac{\partial H_{z}}{\partial r}\right|_{R}= & \left.\frac{\varepsilon_{R}}{\varepsilon_{L}} \frac{\partial H_{z}}{\partial r}\right|_{L} \\
\left.\frac{\partial H_{z}}{\partial \theta}\right|_{R}= & \left.\frac{\partial H_{z}}{\partial \theta}\right|_{L} \\
\left.\frac{\partial^{2} H_{z}}{\partial r^{2}}\right|_{R}= & \left.\frac{\partial^{2} H_{z}}{\partial r^{2}}\right|_{L}+\left.\frac{1}{r}\left(1-\frac{\varepsilon_{R}}{\varepsilon_{L}}\right) \frac{\partial H_{z}}{\partial r}\right|_{L} \\
& +\left.k_{0}^{2}\left(\varepsilon_{L}-\varepsilon_{R}\right) H_{z}\right|_{L} \\
\left.\frac{\partial^{2} H_{z}}{\partial r \partial \theta}\right|_{R}= & \left.\frac{\varepsilon_{R}}{\varepsilon_{L}} \frac{\partial^{2} H_{z}}{\partial r \partial \theta}\right|_{L} \\
\left.\frac{\partial^{2} H_{z}}{\partial \theta^{2}}\right|_{R}= & \left.\frac{\partial^{2} H_{z}}{\partial \theta^{2}}\right|_{L} \\
& \vdots
\end{aligned}
$$

\section{Implementation}

Taking the second-order nine-point formula as an example and following the five steps, the field quantity at each grid point can be expressed as a linear combination of the field quantity $\left.\phi\right|_{(m, n)}$ at point $(m, n)$ and its derivatives. We collect all similar linear equations based on the nine points shown in Fig. 1, including the point $(m, n)$ itself, and express them in a matrix form

$$
\Phi=\mathbf{M} \cdot \mathbf{D}_{(m, n)}
$$

where $\Phi$ is the vector containing the field quantities at the nine points, $\mathbf{M}$ is the matrix of coefficients derived with the five steps, and $\mathbf{D}_{(m, n)}$ is the vector contains the field quantity at the point $(m, n)$ and its derivatives with respect to $x$ or $y$. We can obtain a final set of finite difference formulas by taking inverse operation of (28) as

$$
\mathbf{D}_{(m, n)}=\mathbf{M}^{-1} \cdot \Phi .
$$

The improved finite-difference formulas for the terms $\partial \phi / \partial x, \partial \phi / \partial y, \partial^{2} \phi / \partial x^{2}, \partial^{2} \phi / \partial y^{2}$, and so on in $\mathbf{D}_{(m, n)}$ are then expressed as a linear combination of the field quantities at the nine sampled points. Since the relation between any two points is obtained by fitting the curved step-index interface with the rigorous field expansion technique, the higher-order coefficients in the matrix $\mathbf{M}$ can also be obtained if we include more higher-order derivative terms in each step. It is possible to derive a higher-order scheme with the same procedure we 


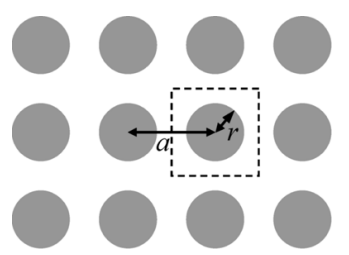

Fig. 2. Cross section of a 2-D photonic crystal with the square lattice where $a$ is the lattice constant and $r$ is the radius of the rods.

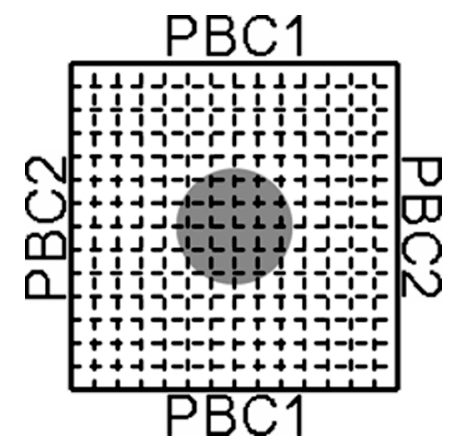

Fig. 3. Unit cell of the photonic crystal with the square lattice and the meshes used in the calculation with the corresponding periodic boundary conditions, $\mathrm{PBC} 1$ and $\mathrm{PBC} 2$.

provided above if more derivative terms and more points are involved. Note that the choice of the sampled points and the derivative terms are not arbitrary. They need to be chosen such that the inverse matrix $\mathbf{M}^{-1}$ in (29) exists.

The cross-section of a 2-D photonic crystal with square lattice is shown in Fig. 2. The unit cell forming the photonic crystal is marked with the dashed lines, where $a$ is the lattice constant and $r$ is the radius of circular rods. We consider only the in-plane propagation and the propagation constant $\beta_{z}$ in the $z$ direction is set to be zero. Due to the periodicity of the structure, we only need to consider one unit cell in the calculation with proper periodic boundary conditions. The unit cell is redrawn with the meshes in the calculation and the periodic boundary conditions in Fig. 3. The periodic boundary conditions PBC1 and PBC2 can be expressed as

$$
\begin{aligned}
& \mathrm{PBC} 1: \phi(x, y+a)=\phi(x, y) \exp \left(-j k_{y} a\right) \\
& \mathrm{PBC} 2: \phi(x+a, y)=\phi(x, y) \exp \left(-j k_{x} a\right)
\end{aligned}
$$

where $k_{x}$ and $k_{y}$ are the wavenumbers in the $x$ and $y$ directions, respectively. Applying our new finite-difference formulas in the Helmholtz's equation for all mesh points in Fig. 3 with periodic boundary conditions given in (30) and (31), a final equation in the matrix form can be obtained as

$$
\left[\mathbf{A}-\left(\frac{\omega}{c}\right)^{2} \mathbf{I}\right] \cdot \widetilde{\Phi}=0
$$

where $\widetilde{\Phi}$ is the vector of the fields at all mesh points, $\mathbf{A}$ is the characteristic matrix with entries formed by the improved finitedifference scheme we derived before with periodic boundary conditions, and $\mathbf{I}$ is the identity matrix. The eigenvalues can be obtained by searching the values of $\omega$ that makes the matrix determinant zero.

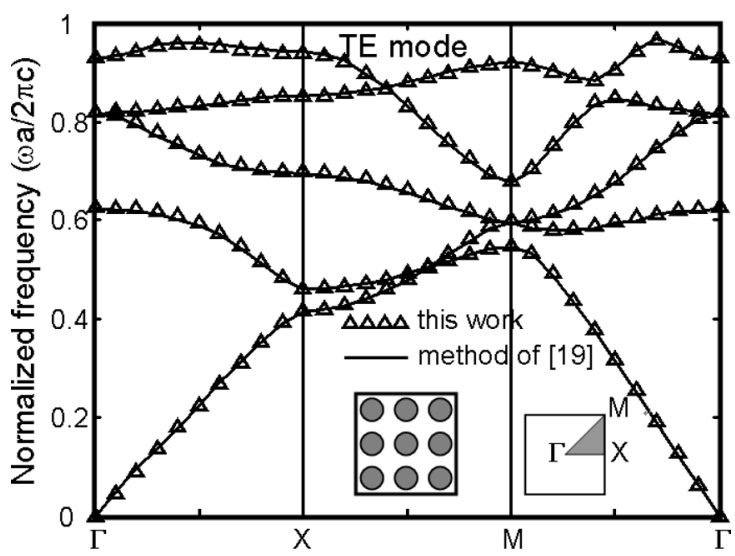

Fig. 4. Calculated band diagram of the TE modes for the 2-D photonic crystal formed by square-arranged alumina rods.

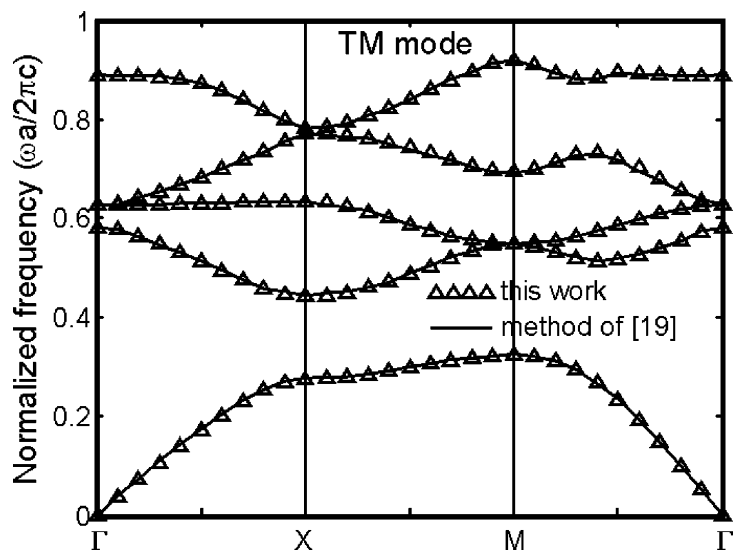

Fig. 5. Calculated band diagram of the TM modes for the 2-D photonic crystal formed by square-arranged alumina rods.

\section{NUMERICAL RESULTS}

\section{A. Assessment of the Formulation}

To validate our improved finite-difference scheme, we choose the previously mentioned 2-D photonic crystal with square lattice as an example. The cross section of the structure under consideration is repeated in the middle inset of Fig. 4. Here, we assume the structure is formed by parallel alumina rods in the air. The relative permittivity of the alumina is $\varepsilon_{\mathrm{Al}}=8.9$ and that of the air is $\varepsilon_{\text {air }}=1$. The radius of the alumina rod is $r=0.2 a$, where $a$ is the lattice constant, as shown in Fig. 2. Each point along the boundary of the first Brillouin zone shown in the right inset in Fig. 4 determines the values of $k_{x}$ and $k_{y}$ that we use in (30) and (31), and thus in (32).

The calculated band diagrams of the TE and TM modes are plotted in Figs. 4 and 5. The band structures marked with up-triangles are the results obtained using our nine-point improved finite-difference scheme derived above with $30 \times 30$ grid points, and the solid lines are the results obtained using the MIT Photonic-Bands package [19] based on the plane-wave expansion method with $128 \times 128$ resolution. They match each other quite well for both TE and TM polarizations.

The computation is performed on a Pentium IV $1.7 \mathrm{GHz}$ personal computer for our case, and the required CPU time is just 


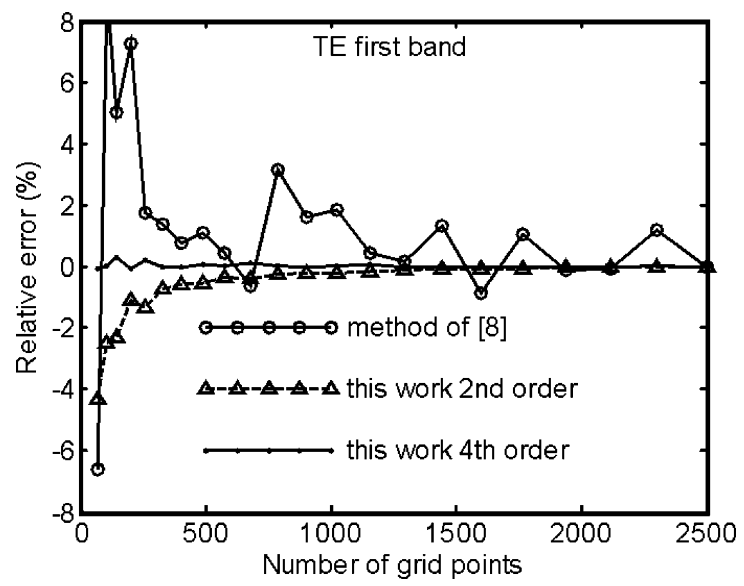

Fig. 6. Convergence property of our methods for the TE first band compared with those by using the methods in [8].

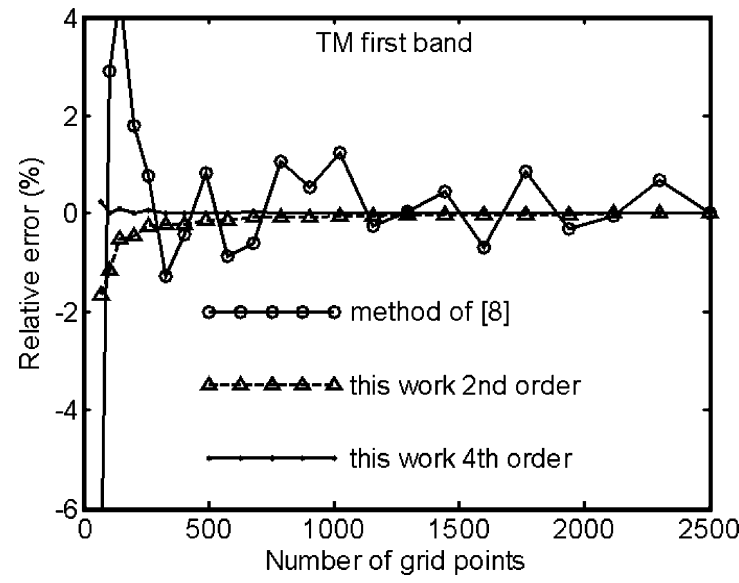

Fig. 7. Convergence property of our methods for the TM first band compared with those by using the methods in [8].

a few minutes. The required CPU time for those results obtained using MIT Photonic-Bands package [19] on a personal computer of the same CPU rating with the linux OS system is also within ten minutes. As indicated in [8], the resultant matrix is quite sparse (quasi-band diagonal), thus it is easy to be implemented with direct $\mathrm{QR}$ procedure and it also reduces the memory requirement.

\section{B. Examination of The Convergence Property}

To examine the efficiency and the convergence behavior of our new scheme, we fix the wave vector $\mathbf{k}$ at the $\mathbf{M}$ point in the first Brillouin zone as in the right inset in Fig. 4, i.e., $\mathbf{k}=$ $(\pi / a, \pi / a, 0)$, and search for the eigen frequencies using different numbers of grid points.

We calculated the results of the four most fundamental bands using our nine-point second-order and 21-point fourth-order schemes, and we also use the schemes provided in [8] to calculate the convergence behavior under the same conditions for comparison and the computed results are also plotted in Figs. 6-9. The solid lines marked with circles in Figs. 6-9 are the results using the scheme in [8], and the dashed lines marked with up-triangles are the results using our nine-point second-order scheme. The solid lines marked with dots in Figs. 6-9 are the results using our 21-point fourth-order

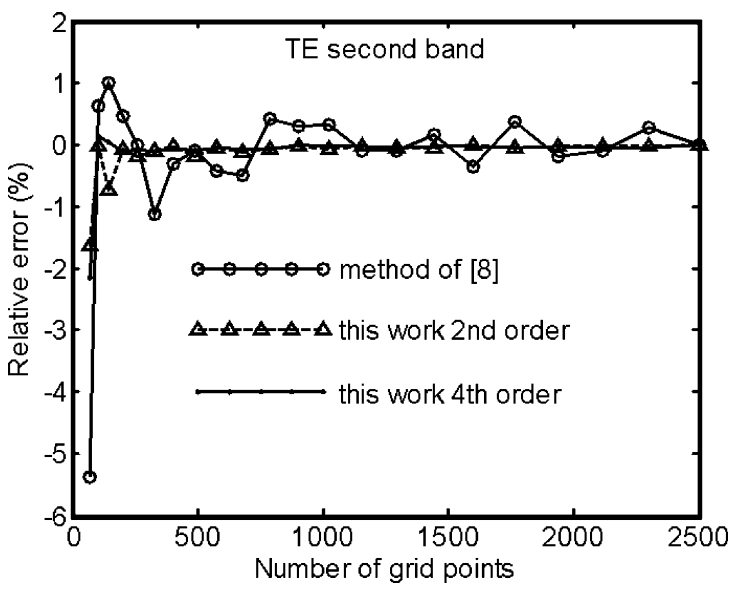

Fig. 8. Convergence property of our methods for the TE second band compared with those by using the methods in [8]

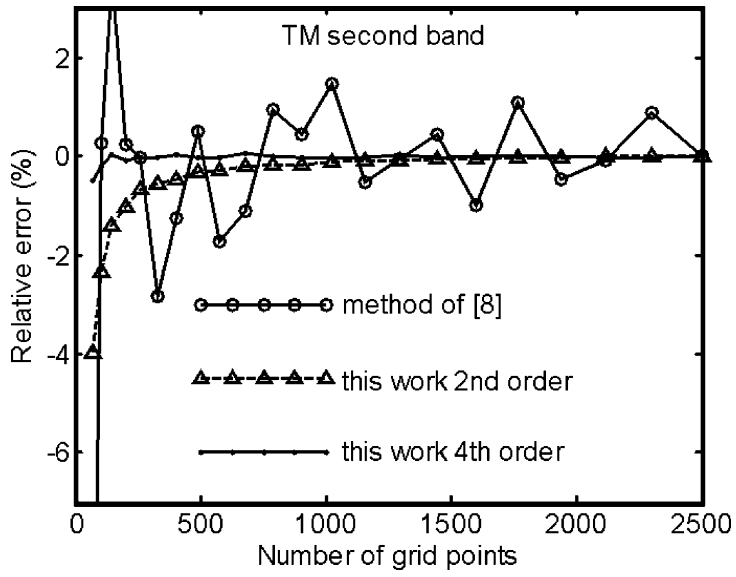

Fig. 9. Convergence property of our method for the TM second band compared with those by using the methods in [8].

scheme. The relative error in Figs. 6-9 refers to the percentage of the difference between the calculated results and a reference results obtained with the number of grid points being 2500 .

It shows that the results for all four bands using our nine-point second-order scheme can achieve a relative error less than about $0.5 \%$, as the number the of total grid points is larger than 1000 . Compared with the results using the method in [8], our method gives better convergence property. Even novel results can be obtained by using our fourth-order scheme. It shows in Figs. 6-9 that all four bands by using fourth-order scheme can achieve a relative error less than about $0.25 \%$, as the number of total grid points is larger than 225 . This means that we need only 15 mesh points in both $x$ and $y$ directions to obtain a result with an acceptable accuracy.

For conventional finite-difference schemes, the contribution of the index-average assumption or the effective index approximation to higher-order terms is hard to quantify. Thus, the difficulty to derive accurate higher-order formulas for these methods lies in how to find the correct coefficients to combine the fields at the sampled points. Since we utilize a rigorous field expansion technique in our derivation, we can accurately obtain the required relations between grid points with interfaces in between for deriving higher-order formulas. The only error in our formulas is from the truncation of the higher-order terms. 


\section{CONCLUSION}

We have derived new finite-difference schemes for solving the band diagram of the 2-D photonic crystals formed by elements with arbitrary shapes. Our results can match very well with those obtained by the plane-wave expansion method, and it is shown that our scheme can improve convergence behavior significantly.

We also demonstrate the convergence behavior of adopting higher-order improved finite-difference scheme by using more terms in the derivation. The result shows that we can further improve the accuracy and reach very good convergence and efficiency.

\section{ACKNOWLEDGMENT}

The authors would like to thank Dr. M. Ho for her helpful discussion on this work.

\section{REFERENCES}

[1] E. Yablonovitch, "Inhibited spontaneous emission in solid-state physics and electronics," Phys. Rev. Lett., vol. 58, no. 20, pp. 2059-2062, May 1987.

[2] S. John, "Strong localization of photons in certain disordered dielectric superlattices," Phys. Rev. Lett., vol. 58, no. 23, pp. 2486-2489, Jun. 1987.

[3] K. M. Ho, C. T. Chan, and C. M. Soukoulis, "Existence of a photonic gap in periodic dielectric structures," Phys. Rev. Lett., vol. 65, no. 25, pp. 3152-3155, Dec. 1990.

[4] M. Plihal and A. A. Maradudin, "Photonic band structure of two-dimensional systems: The triangular lattice," Phys. Rev. B, vol. 44, no. 16, pp. 8565-8571, Oct. 1991.

[5] R. D. Meade, K. D. Brommer, A. M. Rappe, and J. D. Joannopoulos, "Existence of a photonic band gap in two dimensions," Appl. Phys. Lett., vol. 61, no. 4, pp. 495-497, Jul. 1992.

[6] C. T. Chan, Q. L. Yu, and K. M. Ho, "Order-N spectral method for electromagnetic waves," Phys. Rev. B, vol. 51, no. 23, pp. 16635-16642, Jun. 1995.

[7] M. Qiu and S. He, "A nonorthogonal finite-difference time-domain method for computing the band structure of a two-dimensional photonic crystal with dielectric and metallic inclusions," J. Appl. Phys., vol. 87, no. 12, pp. 8268-8275, Jun. 2000.

[8] H. Y. D. Yang, "Finite difference analysis of 2-D photonic crystals," IEEE Trans. Microw. Theory Technol., vol. 44, no. 12, pp. 2688-2695, Dec. 1996.

[9] L. Shen, S. He, and S. Xiao, "A finite-difference eigenvalue algorithm for calculating the band structure of a photonic crystal," Comput. Phys. Commun., vol. 143, no. 3, pp. 213-221, Mar. 2002.

[10] K. Bierwirth, N. Schulz, and F. Arndt, "Finite-difference analysis of rectangular dielectric waveguide structures," IEEE Trans. Microw. Theory Technol., vol. 34, no. 11, pp. 1104-1114, Nov. 1986.

[11] G. R. Hadley and R. E. Smith, "Full-vector waveguide modeling using an iterative finite-difference method with transparent boundary conditions," J. Lightw. Technol., vol. 13, no. 3, pp. 465-469, Mar. 1995.

[12] W. P. Huang, C. L. Xu, S. T. Chu, and S. K. Chaudhuri, "The finitedifference vector beam propagation method: Analysis and assessment," IEEE J. Lightw. Technol., vol. 10, no. 3, pp. 295-305, Mar. 1992.

[13] Z. Zhu and T. G. Brown, "Full-vectorial finite-difference analysis of microstructured optical fibers," Opt. Express, vol. 10, no. 17, pp. 853-864, Aug. 2002.

[14] M. S. Stern, "Semivectorial polarized finite difference method for optical waveguides with arbitrary index profiles," Inst. Elect. Eng. Proc.-J. Optoelectron., vol. 135, no. 1, pp. 56-63, Feb. 1988.

[15] C. Vassallo, "Improvement of finite difference methods for step-index optical waveguides," Inst. Elec. Eng. Proc.-J., vol. 139, no. 2, pp. 137-142, Apr. 1992.

[16] Y.-P. Chiou, Y.-C. Chiang, and H.-C. Chang, "Improved three-point formulas considering the interface conditions in the finite-difference analysis of step-index optical devices," IEEE J. Lightw. Technol., vol. 18, no. 2, pp. 243-251, Feb. 2000.

[17] J. Xia and J. Yu, "New finite-difference scheme for simulations of stepindex waveguides with tilt interfaces," IEEE Photon. Technol. Lett., vol. 15, no. 9, pp. 1237-1239, Sep. 2003.

[18] Y.-C. Chiang, Y.-P. Chiou, and H.-C. Chang, "Improved full-vectorial finite-difference mode solver for optical waveguides with step-index profiles," IEEE J. Lightw. Technol., vol. 20, no. 8, pp. 1609-1618, Aug. 2002.
[19] S. G. Johnson and J. D. Joannopoulos, "The MIT photonic-bands package home page," 2007 [Online]. Available: http://ab-initio.mit.edu/ $\mathrm{mpb} /$

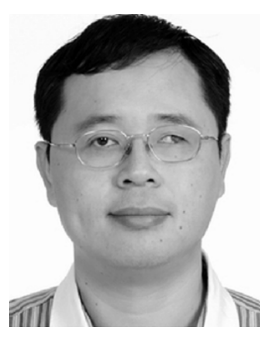

Yen-Chung Chiang (M'06) was born in Hualien, Taiwan, R.O.C., on March 10, 1970. He received the B.S., M.S., and Ph.D. degrees in electrical engineering from National Taiwan University, Taipei, Taiwan, R.O.C., in 1992, 1994, and 2002, respectively. His Ph.D. dissertation was on the mode solver of optical waveguides.

In 2005, he joined the faculty of the Electrical Engineering Department, National Chung-Hsing University, where he is currently an Assistant Professor. From 2002 to 2005 , he was with the VIA Technologies Inc., Taiwan, R.O.C., where his interest was the design of radio-frequency integrated circuits. His current research interests include the numerical analysis techniques for optical or microwave devices and the design of radio-frequency integrated circuits.

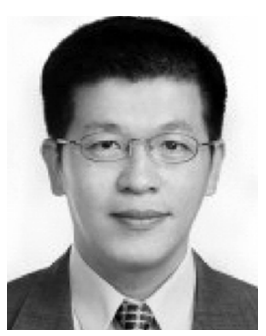

Yih-Peng Chiou (M'03) was born in Taoyuan, Taiwan, R.O.C., in 1969. He received the B.S. and $\mathrm{Ph} . \mathrm{D}$. degrees from National Taiwan University, Taipei, Taiwan, R.O.C., in 1992 and 1998, respectively, both in electrical engineering.

In 2003, he joined the faculty of the Graduate Institute of Electro-Optical Engineering and Department of Electrical Engineering, National Taiwan University, where he is currently an Assistant Professor. From 1999 to 2000, he was with the Taiwan Semiconductor Manufacturing Company, where his interest was the plasma enhanced chemical vapor deposition of dielectric films. From 2001 to 2003, he was with the RSoft Design Group, New York, where his research interests were on the modeling of simulation techniques and developing of photonic computer-aided-design tools. His current research interests include modeling and computer-aided design (CAD) of optoelectronics, which includes photonic crystals, nano structures, waveguide devices, optical fiber devices, light extraction enhancement in LED, display, and solar cell devices, and the development and improvement of numerical techniques in optoelectronics

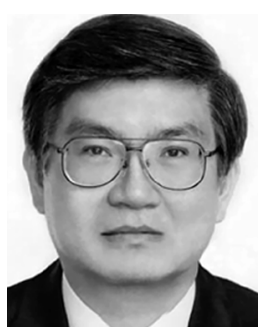

Hung-Chun Chang (S'78-M'83-SM'00) was born in Taipei, Taiwan, R.O.C., on February 8, 1954. He received the B.S. degree from the National Taiwan University, Taipei, Taiwan, R.O.C., in 1976, and the M.S. and Ph.D. degrees from Stanford University, Stanford, CA, in 1980 and 1983, respectively, all in electrical engineering.

In August 1984, he joined the faculty of the Department of Electrical Engineering, National Taiwan University, where he is currently a Professor. From 1978 to 1984 , he was with the Space, Telecommunications, and Radioscience Laboratory, Stanford University. From 1989 to 1991, he served as Vice-Chairman of the Department of Electrical Engineering and, from 1992 to 1998, as Chairman of the newly-established Graduate Institute of Electro-Optical Engineering at the National Taiwan University. He is also with the Graduate Institute of Communication Engineering, National Taiwan University. His current research interests include the theory, design, and application of guided-wave structures and devices for fiber optics, integrated optics, optoelectronics, and microwave- and millimeter-wave circuits.

Dr. Chang is a Member of Sigma Xi, the Phi Tan Phi Scholastic Honor Society, the Chinese Institute of Engineers, the Photonics Society of ChineseAmericans, the Optical Society of America, the Electromagnetics Academy, and the China/SRS (Taipei) National Committee (a Standing Committee member during 1988-1993 and since 2006) of the International Union of Radio Science (URSI). He has been serving as the Institute of Electronics, Information, and Communication Engineers (Japan) Overseas Area Representative in Taipei, since 2002. In 1987, he was among the recipients of the Young Scientists Award at the URSI XXIInd General Assembly. In 1993, he was a recipient of the Distinguished Teaching Award sponsored by the R.O.C., Ministry of Education and in 2004, he received the Merit NSC Research Fellow Award sponsored by the R.O.C., National Science Council. 